\title{
Natural history of ventriculostomy-related infection under appropriate treatment and risk factors of poor outcome: a retrospective study
}

\author{
Roman Mounier, MD, ${ }^{1}$ Ron Birnbaum, MD, ${ }^{1}$ Fabrice Cook, MD, ${ }^{1}$ Paul-Henri Jost, MD, ${ }^{1}$ \\ Mathieu Martin, MD,, Bouziane Aït-Mamar, MD,, Biba Nebbad, MD, ${ }^{1}$ Séverine Couffin, MD, ${ }^{1}$ \\ Françoise Tomberli, MD, ${ }^{1}$ Ryad Djedid, MD, ${ }^{3}$ Gilles Dhonneur, MD, PhD, ${ }^{1}$ and David Lobo, MD1 \\ Departments of ${ }^{1}$ Anesthesia and Surgical Intensive Care, ${ }^{2}$ Microbiology, and ${ }^{3}$ Neurosurgery, Henri Mondor University Hospital of \\ Paris, Paris XII School of Medicine, Créteil, France
}

\begin{abstract}
OBJECTIVE The authors aimed to describe the natural history of ventriculostomy-related infections (VRIs) under appropriate treatment and to assess risk factors for poor outcome.

METHODS All patients older than 18 years in whom an external ventricular drain (EVD) had been implanted and who had developed a VRI requiring treatment were included in this retrospective study. D0 was defined as the first day of antibiotic administration. Clinical and biological parameters were compared each day beginning with D1 and ending with D10 to those of D0. The authors defined D0 in a control group as the day a CSF culture came back positive, without any sign of infection. The authors then searched for poor prognostic factors in the VRI group.

RESULTS Among 567 patients requiring an EVD between January 2007 and October 2017, 39 developed a VRI. Most were monomicrobial infections, and 47 microbes were responsible ( $45 \%$ were gram-positive cocci). Clinical parameters differed significantly from the control group during the first 2 days and then returned to baseline. The CSF parameters differed significantly from the control group for a longer period, returning to baseline after 5 days. CSF sterilization occurred in a median time of 2 days. An intrathecal route or EVD exchange was not associated with a poor outcome. No clinical or biological parameter between D3 and D5 was linked to outcome.
\end{abstract}

CONCLUSIONS Clinical status improved faster than CSF parameters (before and after D5, respectively). Some CSF parameters remained abnormal until D10. Body temperature and microbiological cultures normalized faster than other parameters.

https://thejns.org/doi/abs/10.3171/2018.6.JNS18853

KEYWORDS cerebrospinal fluid; external ventricular drain; natural history; ventriculostomy-related infection; hydrocephalus

$\mathrm{P}$ LACING an external ventricular drain (EVD) exposes one to infectious hazards defined as ventriculostomy-related infections (VRIs). ${ }^{14,19,20}$ The reported incidence of drain-related meningitis ranges from $2 \%$ to $22 \% .14,20$

Little is known about VRI or healthcare-associated meningitis. Factors associated with an increased risk of infection are intraventricular or subarachnoid hemorrhage $(\mathrm{SAH})$, craniotomy, systemic infection, cranial fracture with CSF leakage, and EVD irrigation. ${ }^{14,15,17,20}$ Preventive measures such as intravenous prophylactic antibiotics or antimicrobial-impregnated EVD have been less studied, and results are contradictory. ${ }^{14,27,37,38}$ Optimal treatment has not been the subject of any randomized controlled trials. ${ }^{32}$ In contrast, community-acquired meningitis has been thoroughly documented. The principles of antimicrobial therapy for patients with VRI are generally the same as those for community-acquired meningitis. ${ }^{32}$ Neverthe-

ABBREVIATIONS ESBL = extended-spectrum beta-lactamase; EVD = external ventricular drain; GCS = Glasgow Coma Scale; GNR = gram-negative rods; GOS = Glasgow Outcome Scale; GOSE = GOS-Extended; GPC = gram-positive cocci; GS = Gram staining; IDSA = Infectious Diseases Society of America; SAH = subarachnoid hemorrhage; $\mathrm{VRC}=$ ventriculostomy-related colonization; $\mathrm{VRI}=$ ventriculostomy-related infection; $\mathrm{WBC}=$ white blood cell.

SUBMITTED March 30, 2018. ACCEPTED June 11, 2018.

INCLUDE WHEN CITING Published online November 16, 2018; DOI: 10.3171/2018.6.JNS18853. 
less, some specific features differentiate those infections from community-acquired meningitis. Indeed, clinical monitoring of neurosurgical patients is difficult, and neurosurgery often generates abnormal CSF parameters. ${ }^{19,28}$ Such infections are also harder to treat, both because the responsible bacteria usually have a low susceptibility to antibiotics (with minimal inhibitory concentration $\geq 1$ $\mathrm{mg} / \mathrm{L}$ ), and because the low meningeal inflammation does not allow an efficient diffusion of antibiotics across the blood-brain barrier. ${ }^{21}$

The Infectious Diseases Society of America (IDSA) recommends the monitoring of the response to VRI treatment by using both clinical parameters and CSF cultures. In the event of the absence of any clinical improvement, additional CSF analysis is recommended. Still, the natural history of the infection under treatment is either unknown or little known. In this study, we aimed to describe the natural course of VRI under appropriate antimicrobial therapy and to assess risk factors of poor outcome.

\section{Methods}

We conducted this study in the neurosurgical ICU of a teaching hospital. All patients older than 18 years in whom an EVD had been implanted and who developed VRI and required antibiotic treatment were included. All data were extracted from a neurosurgical ICU and a microbiological database. The institutional ethics committee approved this retrospective analysis.

\section{Exclusion Criteria}

Patients were excluded in the event of the following: 1) EVD colonization or contamination (a positive CSF culture with a good outcome despite no antimicrobial therapy);2) aseptic meningitis (clinical and laboratory pattern of meningitis without any microbiological data at CSF culture); 3) complicated bacterial meningitis (e.g., cerebral abscess); and 4) EVD placement for CSF infection.

\section{Patient Selection}

All patients with EVDs in whom a positive CSF culture was collected between January 2007 and October 2015 were identified and retrospectively analyzed. The patients' charts were reviewed to retrieve demographic, clinical, and laboratory data. Between October 2013 and February 2017, all patients with EVDs who had a positive CSF culture were prospectively analyzed. The following data were recorded: age, sex, underlying neurological condition, indication for EVD placement, length of catheterization, risk factors for infectious disease, systemic and local signs of inflammation, neurological symptoms indicative of secondary meningitis, plasma and CSF laboratory parameters, microbiological data, antimicrobial therapy before development of VRI, antimicrobial prophylaxis during EVD placement, treatment outcome (cured, persistent infection, or death), and Glasgow Outcome Scale (GOS) score at 3 months.

\section{Definitions}

VRI was defined as a positive CSF culture sampled from the EVD, ${ }^{8,15,17,19}$ resulting in a CNS-targeted antibiotic treatment. If common skin flora were isolated, the same strain had to be isolated in at least 2 CSF samples within an interval of less than 96 hours. All strains were held at the microbiological laboratory. The coagulase-negative Staphylococcus strains were assessed by determination of species and antibiotyping. ${ }^{10,19-21,30}$ Poor outcome was defined as a GOS-Extended (GOSE) score $<6 .{ }^{36}$

\section{Time Points of the Analysis}

We analyzed the files of all patients presenting with at least 1 positive CSF culture. Among those, we distinguished 2 groups: those with and those without VRI. The latter was considered a control cohort, suffering from ventriculostomy-related colonization (VRC): despite a positive CSF sample, those patients attained a good outcome without the administration of any antibiotic treatment. In the VRI group, day 0 (D0) was arbitrarily defined as the day an antibiotic treatment was initiated. In order to standardize the analysis, we defined D0 for the control cohort as the day a culture came back positive.

The analysis focused on the first 10 days following D0 (D1-D10). We investigated the evolutionary profiles (each day vs D0) of major CSF meningitis markers (glucose, protein, and leukocyte) among patients with VRI and in the control cohort.

\section{Surgical EVD Procedure}

The standard procedure of EVD placement was applied under sterile conditions in the operating room. No antimicrobial prophylaxis was recommended in our institution, either for placement or during the drainage period. The patient's hair was clipped and the scalp was prepared with standard sterile techniques (povidone-iodine solution). The catheter was subcutaneously tunneled (at least $4 \mathrm{~cm}$ ) and then sutured. Once the nonocclusive dressing was applied, the system remained entirely closed. Wounds were cleaned (povidone-iodine procedure) and dressing was changed every 48 hours. Aseptic technique was applied for CSF sampling at the distal stopcock, and disinfection of the connecting sites with chlorhexidine-ethanol solution was performed when manipulating the system. The EVD was removed under aseptic conditions in the ICU and sent for microbiological analysis. Bactiseal EVDs (Codman, Johnson \& Johnson) were used exclusively.

\section{Procedure When Infection Was Suspected}

When Gram staining (GS) revealed a microorganism, while waiting for culture results to be reported (48 hours later), the following procedure was applied: 1) a neurosurgeon performed a new CSF sampling via the proximal stopcock for microbiological analysis, and 2) the CSF external drainage line was changed.

Antibiotic treatment was initiated for the following reasons: 1) if the patient's clinical condition deteriorated;2) if culture from the proximal stopcock revealed the same strain as the one obtained on the distal sample; or 3) if the daily CSF sampling via the new line had a positive culture with the same strain. The antibiotic regimen and route of administration were left to the discretion of the physicians. 


\section{Microbiological Data}

CSF sampling was performed daily via the drip chamber stopcock. Samples were sent to the laboratory for GS and culture. The drain's tip was vortexed, and the solution was subsequently streaked on blood agar plates. Culture media included 5\% sheep blood agar, chocolate agar (in $5 \% \mathrm{CO}_{2}$ ), tryptic soy agar, MacConkey agar, and Schaedler broth. Inoculated media were incubated at $35^{\circ} \mathrm{C}-37^{\circ} \mathrm{C}$ for 5 days. Bacteria were identified by means of standard biochemical tests. Susceptibility testing was performed by the agar dilution replicate plating method. All cultures were incubated under aerobic and anaerobic conditions.

\section{Statistical Analysis}

We conducted a retrospective longitudinal study to assess the clinical, biological, and microbiological changes related to VRI treatment. We analyzed every patient recorded in our neurosurgical ICU database whose files contained the following keyword term: external ventricular drainage. Details of those having suffered from VRI were recorded day by day, from D0 to D10. Statistical analysis was performed using SPSS statistical software (version 20.0; IBM Corp.). Quantitative values were expressed as the median [25th-75th percentile] and represented as box plots. Each recorded parameter was compared to its D0 value. Wilcoxon signed-rank test analysis was used to depict the changes of the parameters over time. These patients were then compared, day by day, to a control group suffering from VRC. A p value $<0.05$ was considered significant.

\section{Results \\ Patient Population}

Five hundred sixty-seven consecutive patients underwent EVD placement during the study period. One hundred twenty-four patients grew at least 1 positive CSF culture. Among those, 20 were excluded: 8 for complicated bacterial meningitis and 12 for preexisting CNS infection. Sixty-five patients were categorized in the VRC cohort (Fig. 1). Thirty-nine patients (6.9\%) were diagnosed with VRI.

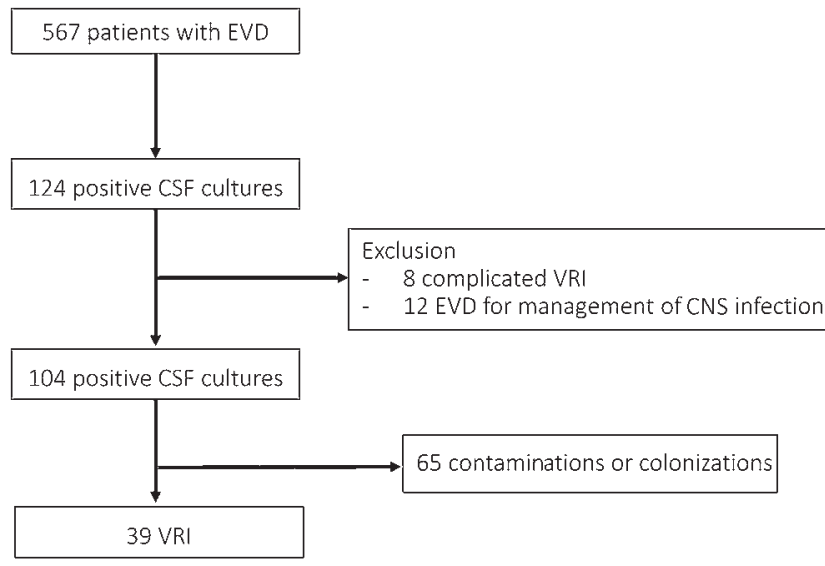

FIG. 1. Flowchart showing the number of EVDs used during the study period and the number of patients excluded and included.
TABLE 1. Baseline characteristics of 39 patients with VRI

\begin{tabular}{|c|c|}
\hline Characteristic & Value \\
\hline Age (yrs) & $53[44-61]$ \\
\hline Sex (female) & $22(56)$ \\
\hline SAPS 2 & $26[15-36]$ \\
\hline \multicolumn{2}{|l|}{ ASA score } \\
\hline 1 & $12(31)$ \\
\hline 2 & $24(62)$ \\
\hline 3 & $3(8)$ \\
\hline Immunodeficiency & $1(3)$ \\
\hline \multicolumn{2}{|l|}{ Cause of admission } \\
\hline $\mathrm{aSAH}$ & $20(51)$ \\
\hline Intracerebral hemorrhage & $4(10)$ \\
\hline $\mathrm{TBI}$ & $3(8)$ \\
\hline Other & $12(31)$ \\
\hline GCS score at admission & $13[7-15]$ \\
\hline Intraventricular hemorrhage & $20(51)$ \\
\hline \multicolumn{2}{|l|}{ Indication for drainage } \\
\hline Acute hydrocephalus & $33(85)$ \\
\hline ATB therapy during EVD placement & $14(36)$ \\
\hline Time from EVD placement to D0 (days) & $12[7-16]$ \\
\hline LOC (days) & $18[11-30]$ \\
\hline ICU stay (days) & 36 [25-49] \\
\hline Delay to EVD exchange (days, $n=20$ ) & $0[0-3]$ \\
\hline ATB therapy duration (days) & $15[13-21]$ \\
\hline Mortality & $9(23)$ \\
\hline VRI-related mortality (\% of dead) & $5(55)$ \\
\hline \multicolumn{2}{|l|}{ GOSE score at 3 mos } \\
\hline $6-8$ & $19(49)$ \\
\hline $4-5$ & $9(23)$ \\
\hline $2-3$ & $2(5)$ \\
\hline 1 & $9(23)$ \\
\hline
\end{tabular}

ASA = American Society of Anesthesiologists; aSAH = aneurysmal SAH; ATB = antibiotic; $L O C=$ length of catheterization; SAPS = Simplified Acute Physiology Score; TBI = traumatic brain injury.

Data are shown as the median [25th-75th percentile] or number (\%), unless otherwise indicated.

Demographic data of these 39 patients are listed in Table 1. Patients were mostly women admitted for SAH, presenting with a median Glasgow Coma Scale (GCS) score of 13. The main indication for CSF drainage was acute hydrocephalus. Patients were catheterized for a median of 18 days, and the time from EVD placement to VRI was 12 days [7-16 days]. The median ICU stay was 36 days. Five of 9 deaths were directly imputed to VRI.

\section{Microbiological Findings}

Forty-seven microbes were responsible, $45 \%$ of which were gram-positive cocci (GPC; Table 2). Staphylococcus epidermidis alone was involved in $26 \%$ of the cases (12/47). Eighty-six percent of GPC were rifampin sensitive, whereas $38 \%$ were clindamycin sensitive and $38 \%$ were sensitive to both drugs. Seven VRIs (18\%) were polymicrobial: 1 VRI with 3 microorganisms (Enterococ- 
TABLE 2. Microbiological findings of the 39 VRIs, antibiotic susceptibility, and concordance with tips cultured

\begin{tabular}{|c|c|c|c|c|c|}
\hline \multirow[b]{2}{*}{ Causative Microorganisms } & \multirow[b]{2}{*}{ No. of Cases } & \multicolumn{3}{|c|}{ Susceptibility of GPC } & \multirow{2}{*}{$\begin{array}{l}\text { No. of EVD Tips } \\
\text { Cultured }\end{array}$} \\
\hline & & Clindamycin & Rifampicin & Both & \\
\hline GPC & & $8(38 \%)$ & $18(86 \%)$ & $8(38 \%)$ & \\
\hline S. epidermidis & 12 & & & & 2 \\
\hline S. aureus & 2 & & & & 1 \\
\hline E. faecalis & 5 & & & & 1 \\
\hline E. faecium & 1 & & & & \\
\hline Streptococcus mitis & 1 & & & & 1 \\
\hline \multicolumn{6}{|l|}{ GNR } \\
\hline E. coli & 3 & & & & \\
\hline K. pneumonia & 5 & & & & 3 \\
\hline K. oxytoca & 3 & & & & 1 \\
\hline$P$. aeruginosa & 5 & & & & \\
\hline E. cloacae & 5 & & & & 2 \\
\hline E. aerogenes & 2 & & & & \\
\hline S. marcescens & 1 & & & & \\
\hline Morganella morganii & 1 & & & & \\
\hline Acinetobacter baumanii & 1 & & & & \\
\hline
\end{tabular}

Only 25 tip cultures were microbiologically analyzed. Tip culture means the number of tip cultures that were positive in culture with the same microorganism as the one causing VRIs. Breakpoint sensitivity from the 2016 guidelines of the European Committee on Antimicrobial Susceptibility Testing.

cus faecalis, Escherichia coli, Enterobacter aerogenes) and 6 VRIs with 2 microorganisms (S. epidermidis-E. faecalis, S. epidermidis-Enterococcus faecium, S. epidermidis-Serratia marcescens, S. epidermidis-E. coli, S. epidermidis-Pseudomonas aeruginosa, and Klebsiella pneumoniae-Enterobacter cloacae).

Only 25 EVD tips were sent to the laboratory and microbiologically analyzed. Eleven of those grew pathogens in culture, yielding concordant microorganisms with those of the VRI.

Initial antimicrobial treatments were all adapted to the causative microorganism. Cefepim, with or without linezolid, was the most common antibiotic administered until documentation of the bacteria. None of the Staphylococcus aureus was methicillin-resistant, in contrast to 83\% of the S. epidermidis. We reported 2 extended-spectrum beta-lactamase (ESBL)-producing E. cloacae, and 1 VIM type metallocarbapenemase-producing $P$. aeruginosa. No beta-lactamase inhibitors were administered.

Patients were intravenously treated for a median time of 15 days [13-21 days]. Twenty patients received 2 [1-3] intrathecal injections (1 every other day; vancomycin [20 $\mathrm{mg}$ ], amikacin [50 $\mathrm{mg}$ ], or gentamicin [8 $\mathrm{mg}]$ ) via the EVD, in combination with the intravenous route, including 1 patient with ESBL-producing E. cloacae, and 1 patient with VIM type carbapenemase-producing $P$. aeruginosa. Six of 9 deceased patients had received intrathecal antibiotics (66\%), compared to 14 of 30 survivors (47\%; $\mathrm{p}=0.45$, OR 2.24, 95\% CI 0.4-16). The median values remained positive until D1 [D0-D3] for GS and D2 [D1D4] for culture. When we compared GS and culture between the survivors and the patients who died, we found no difference except on D8 for GS (1 patient had positive GS per group, $p=0.029)$. The only patient keeping a posi- tive GS and culture on D10 was the one suffering from a carbapenemase-producing Pseudomonas strain. He died with a culture still positive after D10. Two other patients died with a positive GS and culture on D3 and D10. GS and culture remained positive significantly longer for the 3 multidrug-resistant strains (2 ESBL and 1 VIM): 9 [6-9.5] versus $2[1-4]$ days $(p=0.04)$ and $10[9.5-10.5]$ versus 3 $[1.7-5]$ days $(\mathrm{p}=0.005)$.

\section{Clinical Evolution Under Adequate Treatment}

The body temperature of patients with VRI under appropriate treatment evolved quickly, decreasing significantly every day following D0 (Table 3). Only the temperature on D0 was significantly different between patients with VRI and those with VRC ( $p<0.001)$.

On D4 the median GCS became significantly and permanently different from that of D0. In fact, on D4, patients recovered the GCS score from 4 days before D0. The median GCS score was significantly different between groups only on D0 and D1.

\section{Biological Evolution Under Adequate Treatment}

Regarding VRI, our findings are as follows. 1) The white blood cell (WBC) count significantly differed from D0 starting on D2 $(\mathrm{p}=0.01)$. However, this difference did not persist day after day. 2) The median CSF glucose concentrations showed only a little change within the period. 3) The median CSF protein concentration constantly differed from D0 starting on D5. 4) The CSF WBC count constantly differed from D0 starting on D4.

Our comparison between VRC and VRI yielded the following findings. 1) No difference was outlined regarding WBC count between groups at any time. 2) Glucose CSF concentration and CSF WBC count differed signifi- 
TABLE 3. Clinical and biological findings concerning and comparing patients with VRI and VRC

\begin{tabular}{|c|c|c|c|c|c|c|}
\hline Day & Overall Value & VRC & VRI & p Value for VRC vs VRI & No. of Valid Data & $\mathrm{p}$ Value for VRI vs D0 \\
\hline \multicolumn{7}{|c|}{ Body temp $\left({ }^{\circ} \mathrm{C}\right)$} \\
\hline Do -4 & $37.8[37.3 ; 38.4]$ & $37.1[35.2 ; 38.0]$ & $37.8[37.4 ; 38.4]$ & 0.266 & 39 & NA \\
\hline D0 & $38.6[38.0 ; 39.1]$ & $38.0[37.5 ; 38.6]$ & $38.8[38.6 ; 39.3]$ & $<0.001$ & 62 & NA \\
\hline $\mathrm{D} 1$ & $38.2[37.7 ; 38.6]$ & $37.8[37.7 ; 38.2]$ & $38.3[37.8 ; 38.8]$ & 0.076 & 63 & $<0.0001$ \\
\hline D2 & $38.0[37.4 ; 38.6]$ & $37.8[37.4 ; 38.2]$ & $38.1[37.4 ; 38.6]$ & 0.506 & 62 & $<0.0001$ \\
\hline D3 & $38.0[37.4 ; 38.5]$ & $37.8[37.5 ; 38.4]$ & $38.0[37.3 ; 38.6]$ & 0.572 & 62 & 0.0005 \\
\hline D4 & $37.8[37.5 ; 38.2]$ & $37.8[37.5 ; 38.1]$ & $38.0[37.5 ; 38.2]$ & 0.530 & 57 & $<0.0001$ \\
\hline D5 & $37.6[37.2 ; 38.1]$ & $37.8[37.5 ; 38.5]$ & $37.5[37.2 ; 38.0]$ & 0.275 & 56 & $<0.0001$ \\
\hline D6 & $37.6[37.3 ; 38.0]$ & $37.8[37.4 ; 38.0]$ & $37.5[37.2 ; 37.8]$ & 0.115 & 50 & $<0.0001$ \\
\hline D7 & $37.6[37.2 ; 37.9]$ & $37.8[37.5 ; 38.2]$ & $37.6[37.1 ; 37.8]$ & 0.249 & 44 & $<0.0001$ \\
\hline D8 & $37.5[37.3 ; 38.0]$ & $37.9[37.4 ; 38.5]$ & $37.5[37.3 ; 37.8]$ & 0.206 & 41 & $<0.0001$ \\
\hline D9 & $37.5[37.0 ; 37.9]$ & $36.9[35.5 ; 38.3]$ & $37.5[37.1 ; 37.9]$ & 1.000 & 28 & $<0.0001$ \\
\hline D10 & $37.5[37.2 ; 37.8]$ & $37.2[36.5 ; 37.9]$ & $37.5[37.2 ; 37.7]$ & 0.923 & 26 & 0.0001 \\
\hline \multicolumn{7}{|l|}{ GCS score } \\
\hline D0 - 4 & $14[8 ; 15]$ & $11[7 ; 14]$ & $14[9 ; 15]$ & 0.228 & 38 & NA \\
\hline DO & $13[10 ; 15]$ & $14[12 ; 15]$ & $12[7 ; 14]$ & 0.008 & 60 & NA \\
\hline $\mathrm{D} 1$ & $13[10 ; 15]$ & $14[13 ; 15]$ & $13[8 ; 15]$ & 0.037 & 59 & 0.905 \\
\hline D2 & $14[11 ; 15]$ & $14[13 ; 15]$ & $13[9 ; 15]$ & 0.176 & 58 & 0.132 \\
\hline D3 & $14[11 ; 15]$ & $14[12 ; 15]$ & $14[10 ; 15]$ & 0.408 & 57 & 0.052 \\
\hline D4 & $14[11 ; 15]$ & $14[13 ; 15]$ & $14[11 ; 15]$ & 0.473 & 54 & 0.030 \\
\hline D5 & $14[12 ; 15]$ & $14[12 ; 15]$ & $14[11 ; 15]$ & 0.782 & 52 & 0.001 \\
\hline D6 & $14[10 ; 15]$ & $14[13 ; 15]$ & $14[10 ; 15]$ & 0.885 & 46 & 0.003 \\
\hline D7 & $14[10 ; 15]$ & $14[10 ; 15]$ & $14[10 ; 15]$ & 0.736 & 42 & 0.002 \\
\hline D8 & $14[10 ; 15]$ & $14[9 ; 15]$ & $15[12 ; 15]$ & 0.512 & 38 & 0.0001 \\
\hline D9 & $14[11 ; 15]$ & $14[10 ; 14]$ & $14[11 ; 15]$ & 0.336 & 25 & 0.001 \\
\hline D10 & $14[11 ; 15]$ & $8[6 ; 11]$ & $14[11 ; 15]$ & 0.275 & 25 & 0.006 \\
\hline \multicolumn{7}{|l|}{ WBC (G/L) } \\
\hline D0 - 4 & $10.3[7.5 ; 14.9]$ & $7.2[5.0 ; 9.5]$ & $10.3[7.9 ; 15.4]$ & 0.421 & 31 & NA \\
\hline DO & $12.1[9.5 ; 14.9]$ & $12.1[9.2 ; 14.1]$ & $12.5[9.7 ; 15.7]$ & 0.283 & 68 & NA \\
\hline $\mathrm{D} 1$ & $11.9[9.4 ; 14.8]$ & $12.2[9.1 ; 14.9]$ & $11.9[9.9 ; 13.5]$ & 0.921 & 68 & 0.24 \\
\hline D2 & $11.0[8.7 ; 13.4]$ & $11.8[8.3 ; 14.3]$ & $10.1[9.0 ; 12.6]$ & 0.468 & 64 & 0.01 \\
\hline D3 & $10.8[8.6 ; 13.7]$ & $10.8[8.8 ; 14.2]$ & $10.8[8.1 ; 13.3]$ & 0.585 & 59 & 0.02 \\
\hline D4 & $10.8[8.6 ; 14.2]$ & $10.8[8.7 ; 13.9]$ & $11.3[8.3 ; 14.2]$ & 0.863 & 58 & 0.39 \\
\hline D5 & $10.4[8.6 ; 13.6]$ & $10.8[9.3 ; 14.1]$ & $9.6[8.3 ; 13.6]$ & 0.347 & 50 & 0.07 \\
\hline D6 & $10.8[8.1 ; 14.2]$ & $10.8[9.4 ; 14.8]$ & $11.4[7.8 ; 13.9]$ & 0.891 & 37 & 0.13 \\
\hline D7 & $9.9[8.2 ; 12.7]$ & $11.1[8.5 ; 14.4]$ & $9.0[7.1 ; 11.6]$ & 0.099 & 45 & 0.003 \\
\hline D8 & $10.8[6.6 ; 12.8]$ & $10.8[7.1 ; 13.0]$ & $10.4[6.5 ; 12.5]$ & 0.906 & 38 & 0.06 \\
\hline D9 & $9.8[7.8 ; 11.9]$ & $11.9[11.1 ; 19.1]$ & $9.4[6.8 ; 11.6]$ & 0.110 & 18 & 0.24 \\
\hline D10 & $9.0[7.0 ; 10.3]$ & $22.1[14.6 ; 29.7]$ & $9.0[7.0 ; 9.9]$ & 0.551 & 17 & 0.002 \\
\hline \multicolumn{7}{|c|}{ CSF proteins (mg/L) } \\
\hline Do -4 & $322[204 ; 518]$ & $0[0 ; 0]$ & $369[248 ; 521]$ & 0.001 & 33 & NA \\
\hline DO & $548[240 ; 1626]$ & $383[188 ; 1178]$ & 1276 [508; 2344] & 0.001 & 88 & NA \\
\hline D1 & $556[233 ; 1402]$ & $318[150 ; 710]$ & $1287[666 ; 4330]$ & $<0.001$ & 74 & 0.09 \\
\hline D2 & $581[259 ; 1186]$ & $355[127 ; 718]$ & $1042[575 ; 1618]$ & $<0.001$ & 72 & 0.053 \\
\hline D3 & $480[245 ; 1028]$ & $370[230 ; 730]$ & 772 [423; 1202] & 0.022 & 68 & 0.055 \\
\hline D4 & 534 [239; 875] & $410[130 ; 649]$ & 814 [419; 1551] & 0.003 & 63 & 0.16 \\
\hline D5 & 562 [329; 936] & $500[163 ; 648]$ & $740[450 ; 1856]$ & 0.010 & 62 & 0.009 \\
\hline D6 & $400[194 ; 620]$ & $375[128 ; 535]$ & $576[238 ; 1208]$ & 0.046 & 55 & 0.01 \\
\hline $\mathrm{D} 7$ & $444[222 ; 790]$ & $390[178 ; 592]$ & $551[277 ; 1018]$ & 0.209 & 44 & 0.09 \\
\hline
\end{tabular}


» CONTINUED FROM PAGE 1056

TABLE 3. Clinical and biological findings concerning and comparing patients with VRI and VRC

\begin{tabular}{|c|c|c|c|c|c|c|}
\hline Day & Overall Value & VRC & VRI & p Value for VRC vs VRI & No. of Valid Data & $p$ Value for VRI vs DO \\
\hline \multicolumn{7}{|c|}{ CSF proteins (mg/L) (continued) } \\
\hline D8 & $416[192 ; 608]$ & $250[172 ; 528]$ & $482[364 ; 829]$ & 0.066 & 42 & 0.02 \\
\hline D9 & $411[312 ; 973]$ & $0[0 ; 0]$ & $442[318 ; 1024]$ & 0.023 & 21 & 0.01 \\
\hline D10 & $278[134 ; 628]$ & $0[0 ; 0]$ & $280[230 ; 718]$ & 0.034 & 19 & 0.02 \\
\hline \multicolumn{7}{|c|}{ CSF glucose (mmol/L) } \\
\hline D0 - 4 & $4.1[3.7 ; 4.6]$ & $4.0[3.8 ; 4.4]$ & $4.1[3.7 ; 4.6]$ & 0.934 & 33 & NA \\
\hline D0 & $4.0[2.6 ; 5.0]$ & $4.6[3.8 ; 5.2]$ & $2.8[0.3 ; 3.6]$ & $<0.001$ & 87 & NA \\
\hline $\mathrm{D} 1$ & $4.0[3.0 ; 4.9]$ & $4.4[3.8 ; 5.0]$ & $2.7[0.6 ; 3.7]$ & $<0.001$ & 74 & 0.30 \\
\hline $\mathrm{D} 2$ & $4.0[3.2 ; 4.6]$ & $4.4[3.8 ; 5.0]$ & $2.9[1.5 ; 3.8]$ & $<0.001$ & 72 & 0.39 \\
\hline D3 & $3.8[3.0 ; 4.7]$ & $4.3[3.6 ; 5.1]$ & $2.9[2.2 ; 3.4]$ & $<0.001$ & 66 & 0.72 \\
\hline D4 & $4.0[3.0 ; 4.6]$ & $4.5[3.9 ; 5.0]$ & $2.7[1.5 ; 3.4]$ & $<0.001$ & 62 & 0.57 \\
\hline D5 & $4.0[2.8 ; 4.6]$ & $4.5[4.0 ; 5.2]$ & $2.8[1.6 ; 3.6]$ & $<0.001$ & 62 & 0.61 \\
\hline D6 & $4.0[3.0 ; 4.6]$ & $4.3[4.0 ; 5.0]$ & $3.0[2.2 ; 3.8]$ & $<0.001$ & 55 & 0.53 \\
\hline D7 & $4.0[3.1 ; 4.9]$ & $4.6[4.0 ; 5.2]$ & $3.1[2.2 ; 3.7]$ & $<0.001$ & 44 & 0.54 \\
\hline D8 & $3.9[3.3 ; 4.4]$ & $4.2[3.6 ; 5.0]$ & $3.6[2.3 ; 3.9]$ & 0.004 & 42 & 0.21 \\
\hline D9 & $3.2[2.4 ; 3.9]$ & $4.1[4.0 ; 4.2]$ & $3.0[2.3 ; 3.8]$ & 0.208 & 21 & 0.08 \\
\hline D10 & $4.0[2.5 ; 4.6]$ & $5.3[5.0 ; 5.6]$ & $3.8[2.4 ; 4.2]$ & 0.054 & 19 & 0.28 \\
\hline \multicolumn{7}{|c|}{ CSF WBC $\left(/ \mathrm{mm}^{3}\right)$} \\
\hline Do - 4 & $5.5[1.2 ; 38.2]$ & $29.5[14.5 ; 54.5]$ & $5.0[1.2 ; 35.8]$ & 0.450 & 34 & NA \\
\hline D0 & $19.0[4.0 ; 410.0]$ & $8.0[2.0 ; 30.0]$ & $337.5[55.0 ; 900.0]$ & $<0.001$ & 93 & NA \\
\hline D1 & $12.0[2.0 ; 104.0]$ & $8.0[1.0 ; 22.0]$ & $174.5[8.5 ; 2800.0]$ & $<0.001$ & 77 & 0.12 \\
\hline D2 & $17.0[5.0 ; 321.5]$ & $6.0[2.0 ; 40.0]$ & $250.0[41.8 ; 687.5]$ & $<0.001$ & 71 & 0.97 \\
\hline D3 & $16.0[3.8 ; 78.2]$ & $6.0[2.5 ; 45.5]$ & $44.0[11.0 ; 126.0]$ & 0.012 & 72 & 0.16 \\
\hline D4 & $8.0[3.0 ; 71.0]$ & $4.0[2.0 ; 29.0]$ & $40.0[7.5 ; 175.0]$ & 0.002 & 68 & 0.04 \\
\hline D5 & $16.5[3.8 ; 71.8]$ & $8.0[2.0 ; 26.5]$ & $58.0[12.8 ; 213.0]$ & $<0.001$ & 64 & 0.02 \\
\hline D6 & $7.5[1.0 ; 45.2]$ & $3.0[1.0 ; 25.5]$ & $18.0[4.2 ; 60.2]$ & 0.063 & 56 & 0.001 \\
\hline D7 & $4.5[1.0 ; 31.2]$ & $2.5[1.0 ; 20.5]$ & $10.0[3.2 ; 57.2]$ & 0.046 & 48 & 0.001 \\
\hline D8 & $5.5[1.0 ; 20.5]$ & $4.0[1.0 ; 12.5]$ & $8.0[5.0 ; 30.0]$ & 0.008 & 44 & 0.0003 \\
\hline D9 & 9.5 [2.0; 27.8] & $1.0[1.0 ; 1.0]$ & $11.5[3.5 ; 42.2]$ & 0.051 & 22 & 0.0004 \\
\hline D10 & $3.0[1.5 ; 8.0]$ & $0.5[0.2 ; 0.8]$ & $3.0[2.0 ; 8.0]$ & 0.071 & 19 & Unknown \\
\hline
\end{tabular}

D0 - 4 = 4 days before D0 (represents the value of reference before the infection); NA = not applicable; temp = temperature.

Boldface type indicates statistical significance. Values are expressed as the median [25th percentile; 75th percentile]. D0 in patients with VRC refers to the day when a positive culture was found.

cantly between groups from D0 to D8. 3) The protein CSF concentration remained significantly different between groups during the entire analysis (Fig. 2, Table 3).

\section{Association With Mortality or Poor Outcome}

Intrathecal infusion of antibiotics was not associated with either poor outcome (GOSE: 7 [4-8] vs 5 [1-6], $\mathrm{p}=$ $0.16)$ or mortality $(\mathrm{p}=0.45)$. The EVD was exchanged in 20 of 39 patients, but this procedure was not associated with either poor outcome (GOSE: 5 [2-7] vs 5 [4-6], $\mathrm{p}=$ $0.61)$ or mortality $(\mathrm{p}=0.95)$. No difference was evidenced regarding time to CSF sterilization between the survivors and the patients who died.

\section{Assessment of Biological Values as Markers of VRI Outcome}

By using extreme values of biological parameters with- in D3 and D5, we tried to correlate our parameters with poor outcome. None were different between the patients who died and the survivors (Fig. 3). No parameter showed a discrimination threshold with a sufficient sensitivity/specificity on a receiver operating characteristic curve (data not shown).

\section{Discussion}

The IDSA's latest guidelines recommend monitoring of the response to VRI treatment. ${ }^{32}$ Still, until now and to our knowledge, no study has yet focused on the natural course of VRI under treatment. ${ }^{32}$ Understanding the normal evolution of clinical and biological parameters under treatment would certainly improve the management of VRI. ${ }^{32}$ We therefore aimed to describe these evolutionary patterns. In addition, we tried to assess factors associated with poor outcome. Our study outlines the fact that clini- 

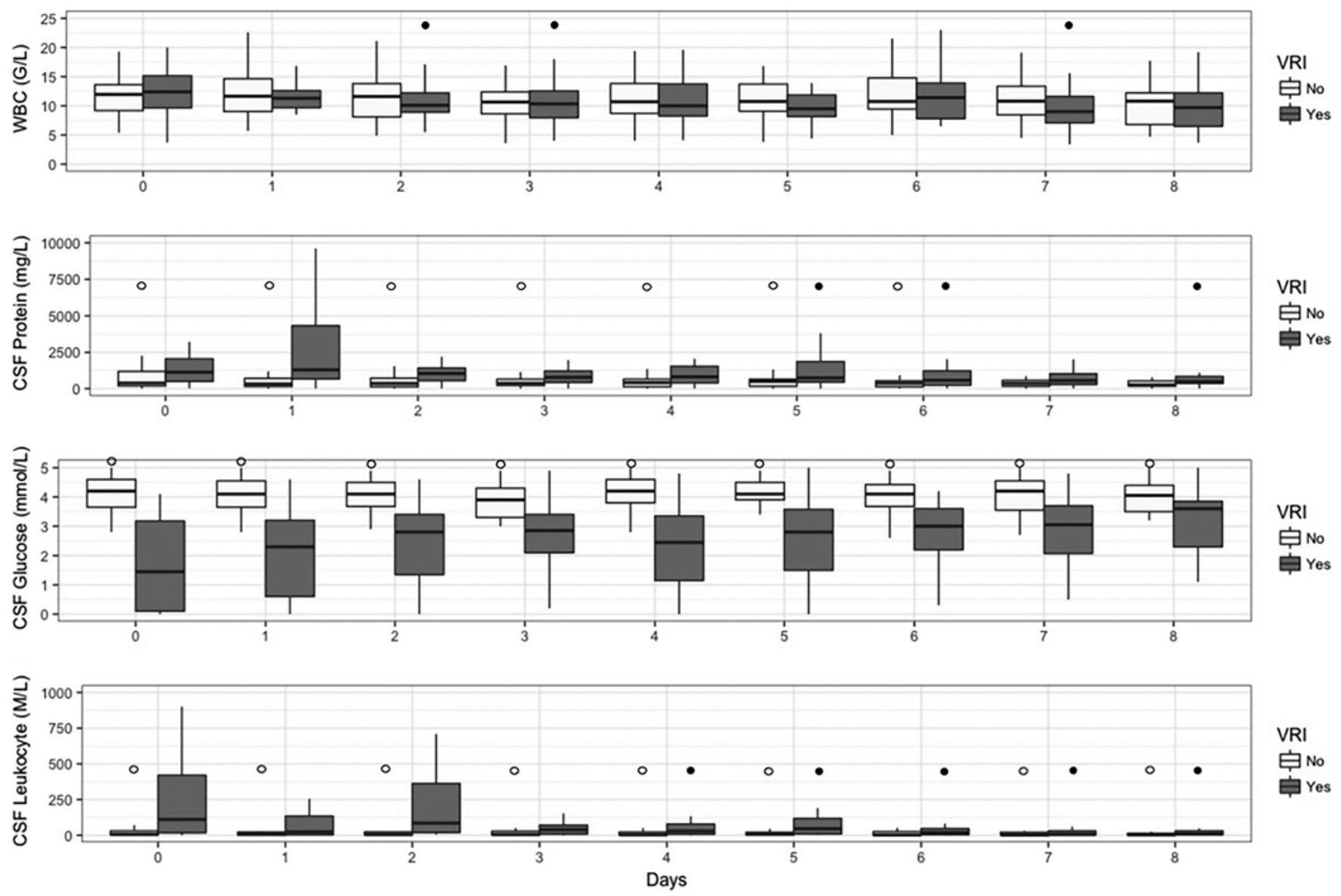

FIG. 2. Box plots showing changes in biological parameters from D0 to D8. Within patients with VRI, comparison was made versus D0 by using the Wilcoxon signed-rank test on the matched data available. Solid circles signify $p<0.05$ versus D0 value. Open circles signify $p<0.05$ when comparing patients with VRI and those with VRC day by day. To improve readability, we have chosen to present graphic data as box plots without outliers. D0 = day of VRI diagnosis.

cal parameters were the earliest to significantly change, within the first days after treatment initiation (D1 for body temperature, D4 for the GCS). Protein in CSF significantly changed on D5, and WBC count in CSF significantly changed on D4. GS and CSF culture remained positive until D1 and D2, respectively. However, none of these parameters allowed sufficient discrimination between a good and a poor outcome.

We still do not know much about the natural course of VRI. Community-acquired meningitis has been more studied, but knowledge remains poor. Without easy access to CSF, physicians have to base their monitoring on clinical parameters. Literature on community-acquired meningitis reports that neurological symptoms improve promptly within the first 48 hours after initiation of therapy, ${ }^{16}$ whereas body temperature normalizes within 4 days. ${ }^{16}$ Regarding patients suffering from VRI, the IDSA guidelines recommend that physicians should evaluate the extent of their response to treatment by using clinical parameters. ${ }^{32}$ Interestingly, temperature improves quickly (within the first 24 hours of treatment) in VRI, although hyperthermia is a common symptom during any neurological disorder. This is corroborated by the fact that temperature was different between patients with VRI and those with VRC only on
D0. In VRI, GCS scores improved more slowly, making this parameter less interesting to monitor for improvement. Four days were necessary to observe a significant improvement, probably because in healthcare-associated meningitis, the neurological disorder due to the infection overlaps that of the underlying neurological disease. This finding is important because it is recommended that the therapeutic strategy be changed (repeat spinal tap, switch antibiotics) $)^{31,34}$ in the absence of a GCS score improvement on D2.

In the absence of any clinical improvement, the IDSA recommends that additional CSF analysis be performed to verify the normalization of CSF parameters and the negativity of cultures. In our study, cultures remained positive until D2 in median, whatever the route of treatment or the outcome. The result of the culture, in conjunction with the lack of improvement of the CSF parameters, can surely be considered alarming. In this study, we outline that CSF abnormalities persist for a long time-therefore, monitoring CSF glucose concentration does not seem relevant because it remained significantly abnormal within the whole period of monitoring; CSF protein concentration and WBC count significantly and consistently improved at approximately D4-5, making those 2 parameters much more interesting in order to monitor the course of VRI 

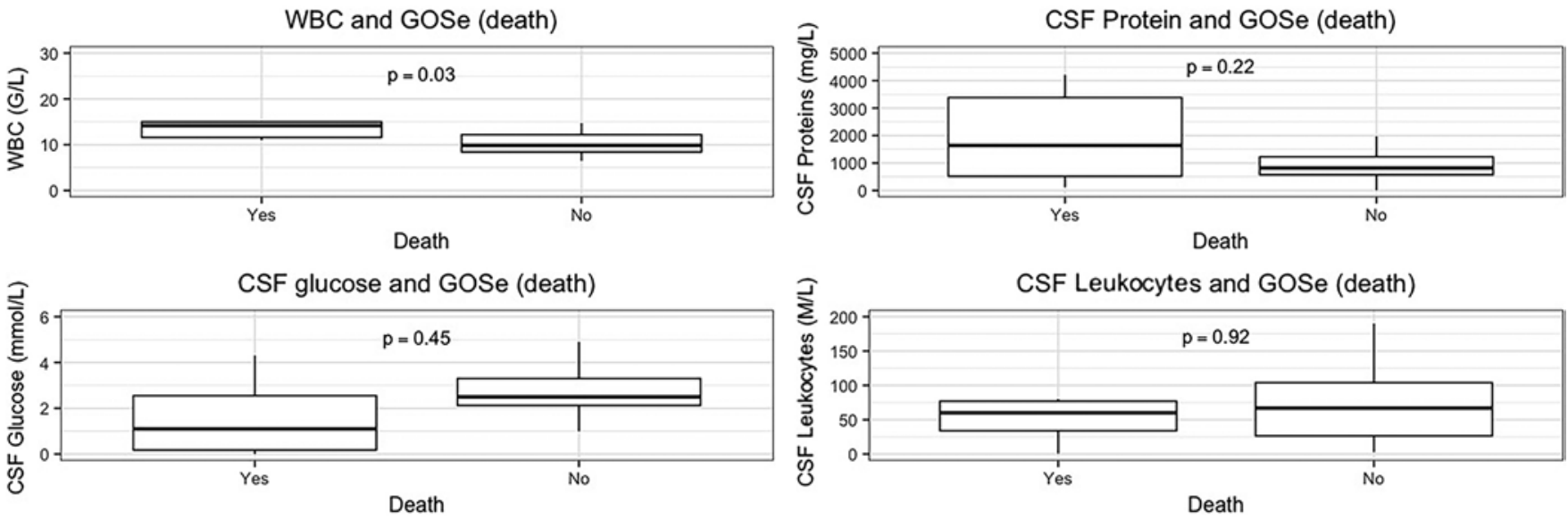

WBC and GOSe (cut-off 6)
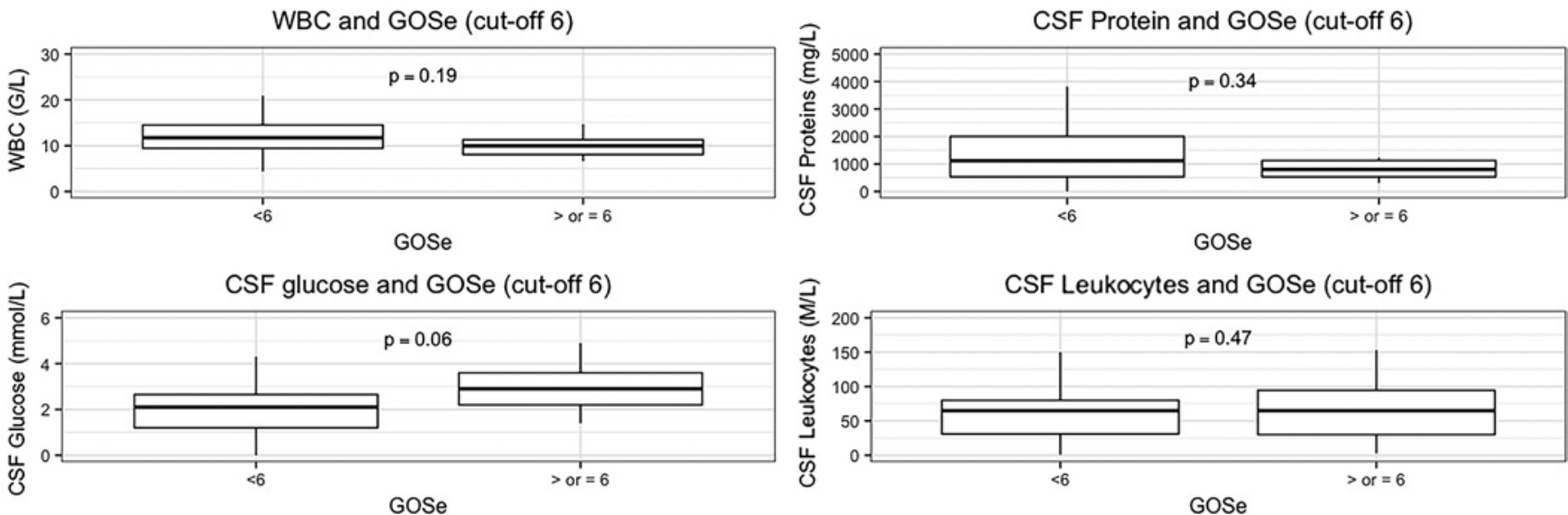

FIG. 3. Box plots showing extreme biological values within D3 and D5 comparing different groups: alive and dead patients; GOSE $<6$ and GOSE $\geq 6$.

under treatment. 5,14,19,22,29 The WBC count still fluctuated over time and, in a more global view, its value tends to be nonspecific in the ICU. In light of these points, regarding patients not improving rapidly, we would tend to check for culture sterilization on D3 and for CSF parameters (except for glucose in CSF) on D5. Still, in our study this attitude did not allow for outcome discrimination. However, the latter could result either from a lack of power of the study or from an increasing number of missing values reported after D0.

Regarding community-acquired meningitis under treatment, CSF usually becomes sterile within the first 24 hours of treatment. ${ }^{12,16,26}$ In our patients with VRI this was clearly not the case: cultures remained positive until D2. Only a limited amount of data are reported in the literature. Both in VRI and community-acquired meningitis, time to CSF sterilization differs according to the causative pathogen, ${ }^{16} \mathrm{a}$ higher minimal inhibitory concentration to antibiotics for nosocomial bacteria, and the presence of a foreign body (the EVD). ${ }^{3,18,21}$ Time to microbiological sterilization was significantly longer for multidrug-resistant bacteria $(\mathrm{p}=$ 0.005 and $p=0.04$ for culture and GS, respectively), as reported in the literature, ${ }^{13}$ but without any impact on outcome.

Regarding risk factors for unfavorable outcome, no study has yet attempted to predict the evolutionary pat- tern of the initial clinical, biological, and microbiological parameters. The literature generally reports their initial values, but does not study the parameters further. ${ }^{23,33} \mathrm{In}$ our work, we describe the natural course of clinical and biological parameters. Unlike others, we report that the GCS score between D3 and D5 is not linked to outcome. Initial WBC count in CSF has previously been associated with outcome. ${ }^{33}$ In our study, none of the parameters were linked to outcome, but the comparison was difficult because we assessed the evolution of the parameters rather than their initial value. The rare studies that evaluate a difference in antimicrobial treatment of meningitis did not analyze the change in CSF parameters. Peltola et al. reported some abnormal biochemistry on D1 for all the CSF parameters, persisting on $\mathrm{D} 4 .{ }^{26}$ Although microbiological analysis requires 2 days, it seems more appropriate to evaluate improvement with this parameter rather than with CSF biochemistry, which takes a longer time to normalize.

Surprisingly, both intrathecal administration of antimicrobials and EVD exchange were not associated with a different outcome. A potential link could have been outlined if more patients had been included. We conducted a retrospective study during which those 2 therapeutic strategies were left at the discretion of the physician in charge of the patient. Efficacy and safety of the intrathecal route of administration has not yet been demonstrated in con- 
trolled trials and there is insufficient evidence to recommend its use. Intrathecal administration could be proposed in the event of infections caused by resistant organisms that are difficult to eradicate with intravenous antimicrobial therapy alone, or when the infection responds poorly to systemic therapy. ${ }^{32}$ This was the case for some of our resistant microorganisms but not all, and despite that, there was no significant difference regarding the prognosis. EVD exchange depends on the patient's condition, the neurosurgeon's preferences, and the kind of microorganism (e.g., infection with S. epidermidis could in some cases be treated without removing the material). ${ }^{4,9}$

Gram-negative rods (GNR) were predominant (26/47, $55.3 \%$ ) in our study, whereas most authors report a predominance of GPC. ${ }^{11,14,24,27,35}$ Some authors, however, do relate a predominance of GNR. ${ }^{6,715}$ Different explanations probably coexist: 1) a difference in epidemiology, depending on the country or ICU; and 2) a different antibiotic regimen including prophylaxis. In our center we did not recommend any intravenous prophylaxis because it was not recommended by guidelines until 2017. ${ }^{14,32}$ Nevertheless, $36 \%$ of our patients still received this prophylaxis. In any case, the median duration of drainage before VRI diagnosis was 12 days [7-16 days], and it is quite unlikely that periprocedural intravenous prophylaxis would maintain some activity for that long. We used Bactiseal EVDs (Codman, Johnson \& Johnson) that diffuse a combination of clindamycin and rifampicin. These antibiotics are essentially active against GPC; ex vivo data state that protection lasts for 56 days ${ }^{1,2}$ and one clinical study reports activity for up to 127 days. ${ }^{25}$ This EVD could therefore have modified our microbial epidemiology, but this seems unlikely because our GPC were $86 \%$ rifampicin susceptible, $38 \%$ clindamycin susceptible, and $38 \%$ were susceptible to both drugs. Surprisingly, few bacteria were resistant, leading to the question of the duration of antimicrobial protection or to a biofilm infection., ${ }^{3,18}$

\section{Study Limitations}

Our study obviously contains some limitations. It is retrospective (the low incidence of VRI requires this design), but a small number of patients were prospectively included $(n=7)$ and added to the cohort already published..$^{19}$ The longitudinal design requiring systematic sampling and data acquisition may have reduced the risk of major bias. Unfortunately, our work lacks power to discriminate a good from a poor outcome. We identified 39 patients with VRI among 567 patients in the course of 8 years, which represents the largest series published until now. We are not aware of another study reporting the natural course of meningitis under treatment.

\section{Conclusions}

We report a consecutive cohort of 39 patients with VRI (6.9\% of the screened population) and describe the clinical, biological, and microbiological pattern of VRI under treatment. This description could help the physician in the management of VRI and allows a better appreciation of the recommendations. We reported a quick normalization of body temperature (D1), followed by the sterilization of culture (D2), and then by improvement of the GCS score
(D4). The CSF glucose concentration seems useless, in contrast to the CSF protein and WBC count, which both show a more consistent and significant switch on D4-5. No parameters were linked to the prognosis.

\section{References}

1. Bayston R, Grove N, Siegel J, Lawellin D, Barsham S: Prevention of hydrocephalus shunt catheter colonisation in vitro by impregnation with antimicrobials. J Neurol Neurosurg Psychiatry 52:605-609, 1989

2. Bayston R, Lambert E: Duration of protective activity of cerebrospinal fluid shunt catheters impregnated with antimicrobial agents to prevent bacterial catheter-related infection. J Neurosurg 87:247-251, 1997

3. Braxton EE Jr, Ehrlich GD, Hall-Stoodley L, Stoodley P, Veeh R, Fux C, et al: Role of biofilms in neurosurgical device-related infections. Neurosurg Rev 28:249-255, 2005

4. Brown EM, Edwards RJ, Pople IK: Conservative management of patients with cerebrospinal fluid shunt infections. Neurosurgery 58:657-665, 2006

5. Carbonnelle E: [Laboratory diagnosis of bacterial meningitis: usefulness of various tests for the determination of the etiological agent.] Med Mal Infect 39:581-605, 2009 (Fr)

6. Chatzi M, Karvouniaris M, Makris D, Tsimitrea E, Gatos $\mathrm{C}$, Tasiou A, et al: Bundle of measures for external cerebral ventricular drainage-associated ventriculitis. Crit Care Med 42:66-73, 2014

7. Chi H, Chang KY, Chang HC, Chiu NC, Huang FY: Infections associated with indwelling ventriculostomy catheters in a teaching hospital. Int J Infect Dis 14:e216-e219, 2010

8. Clark WC, Muhlbauer MS, Lowrey R, Hartman M, Ray MW, Watridge CB: Complications of intracranial pressure monitoring in trauma patients. Neurosurgery 25:20-24, 1989

9. Conen A, Walti LN, Merlo A, Fluckiger U, Battegay M, Trampuz A: Characteristics and treatment outcome of cerebrospinal fluid shunt-associated infections in adults: a retrospective analysis over an 11-year period. Clin Infect Dis 47:73-82, 2008

10. Couffin S, Lobo D, Cook F, Jost PH, Bitot V, Birnbaum R, et al: Coagulase-negative staphylococci are associated to the mild inflammatory pattern of healthcare-associated meningitis: a retrospective study. Eur J Clin Microbiol Infect Dis 37:755-763, 2018

11. Harrop JS, Sharan AD, Ratliff J, Prasad S, Jabbour P, Evans JJ, et al: Impact of a standardized protocol and antibioticimpregnated catheters on ventriculostomy infection rates in cerebrovascular patients. Neurosurgery 67:187-191, 2010

12. Kanegaye JT, Soliemanzadeh P, Bradley JS: Lumbar puncture in pediatric bacterial meningitis: defining the time interval for recovery of cerebrospinal fluid pathogens after parenteral antibiotic pretreatment. Pediatrics 108:1169-1174, 2001

13. Karaiskos I, Galani L, Baziaka F, Giamarellou H: Intraventricular and intrathecal colistin as the last therapeutic resort for the treatment of multidrug-resistant and extensively drug-resistant Acinetobacter baumannii ventriculitis and meningitis: a literature review. Int J Antimicrob Agents 41:499-508, 2013

14. Lozier AP, Sciacca RR, Romagnoli MF, Connolly ES Jr: Ventriculostomy-related infections: a critical review of the literature. Neurosurgery 51:170-182, 2002

15. Lyke KE, Obasanjo OO, Williams MA, O’Brien M, Chotani R, Perl TM: Ventriculitis complicating use of intraventricular catheters in adult neurosurgical patients. Clin Infect Dis 33:2028-2033, 2001

16. Martin E, Hohl P, Guggi T, Kayser FH, Fernex M: Short course single daily ceftriaxone monotherapy for acute bacterial meningitis in children: results of a Swiss multicenter study. Part I: clinical results. Infection 18:70-77, 1990 
17. Mayhall CG, Archer NH, Lamb VA, Spadora AC, Baggett JW, Ward JD, et al: Ventriculostomy-related infections. A prospective epidemiologic study. N Engl J Med 310:553559, 1984

18. Mounier R, Kapandji N, Birnbaum R, Cook F, Rodriguez C, Nebbad B, et al: Biofilm-associated infection: the hidden face of cerebrospinal fluid shunt malfunction. Acta Neurochir (Wien) 158:2321-2324, 2016

19. Mounier R, Lobo D, Cook F, Fratani A, Attias A, Martin M, et al: Clinical, biological, and microbiological pattern associated with ventriculostomy-related infection: a retrospective longitudinal study. Acta Neurochir (Wien) 157:2209-2217, 2015

20. Mounier R, Lobo D, Cook F, Martin M, Attias A, Aït-Mamar $\mathrm{B}$, et al: From the skin to the brain: pathophysiology of colonization and infection of external ventricular drain, a prospective observational study. PLoS One 10:e0142320, 2015

21. Mounier R, Lobo D, Hulin A, Nebbad B, Cook F, Dhonneur G: Is first-line vancomycin still the best option to treat staphylococcus health care-associated meningitis? World Neurosurg 99:812.e1-812.e5, 2017

22. Muttaiyah S, Ritchie S, Upton A, Roberts S: Clinical parameters do not predict infection in patients with external ventricular drains: a retrospective observational study of daily cerebrospinal fluid analysis. J Med Microbiol 57:207-209, 2008

23. Nathan N, Borel T, Djibo A, Evans D, Djibo S, Corty JF, et al: Ceftriaxone as effective as long-acting chloramphenicol in short-course treatment of meningococcal meningitis during epidemics: a randomised non-inferiority study. Lancet 366:308-313, 2005

24. Park J, Choi YJ, Ohk B, Chang HH: Cerebrospinal fluid leak at percutaneous exit of ventricular catheter as a crucial risk factor for external ventricular drainage-related infection in adult neurosurgical patients. World Neurosurg 109:e398e403, 2018

25. Pattavilakom A, Kotasnas D, Korman TM, Xenos C, Danks A: Duration of in vivo antimicrobial activity of antibioticimpregnated cerebrospinal fluid catheters. Neurosurgery 58:930-935, 2006

26. Peltola H, Anttila M, Renkonen OV: Randomised comparison of chloramphenicol, ampicillin, cefotaxime, and ceftriaxone for childhood bacterial meningitis. Lancet 1:1281-1287, 1989

27. Pople I, Poon W, Assaker R, Mathieu D, Iantosca M, Wang E, et al: Comparison of infection rate with the use of antibioticimpregnated vs standard extraventricular drainage devices: a prospective, randomized controlled trial. Neurosurgery 71:6-13, 2012

28. Schade RP, Schinkel J, Roelandse FWC, Geskus RB, Visser LG, van Dijk JMC, et al: Lack of value of routine analysis of cerebrospinal fluid for prediction and diagnosis of external drainage-related bacterial meningitis. J Neurosurg 104:101108, 2006 (Erratum in J Neurosurg 106:941, 2007)

29. Scheithauer S, Bürgel U, Ryang YM, Haase G, Schiefer J, Koch S, et al: Prospective surveillance of drain associated meningitis/ventriculitis in a neurosurgery and neurological intensive care unit. J Neurol Neurosurg Psychiatry 80:1381-1385, 2009
30. Sharma M, Riederer K, Johnson LB, Khatib R: Molecular analysis of coagulase-negative Staphylococcus isolates from blood cultures: prevalence of genotypic variation and polyclonal bacteremia. Clin Infect Dis 33:1317-1323, 2001

31. Tunkel AR, Hartman BJ, Kaplan SL, Kaufman BA, Roos KL, Scheld WM, et al: Practice guidelines for the management of bacterial meningitis. Clin Infect Dis 39:1267-1284, 2004

32. Tunkel AR, Hasbun R, Bhimraj A, Byers K, Kaplan SL, Michael Scheld W, et al: 2017 Infectious Diseases Society of America's Clinical Practice Guidelines for Healthcare-Associated Ventriculitis and Meningitis. Clin Infect Dis [epub ahead of print], 2017

33. van de Beek D, de Gans J, Spanjaard L, Weisfelt M, Reitsma JB, Vermeulen M: Clinical features and prognostic factors in adults with bacterial meningitis. N Engl J Med 351:18491859,2004

34. van de Beek D, de Gans J, Tunkel AR, Wijdicks EFM: Community-acquired bacterial meningitis in adults. $\mathbf{N ~ E n g l ~}$ J Med 354:44-53, 2006

35. Walti LN, Conen A, Coward J, Jost GF, Trampuz A: Characteristics of infections associated with external ventricular drains of cerebrospinal fluid. J Infect 66:424-431, 2013

36. Wilson JT, Pettigrew LE, Teasdale GM: Structured interviews for the Glasgow Outcome Scale and the extended Glasgow Outcome Scale: guidelines for their use. J Neurotrauma 15:573-585, 1998

37. Wong GKC, Poon WS, Ng SCP, Ip M: The impact of ventricular catheter impregnated with antimicrobial agents on infections in patients with ventricular catheter: interim report. Acta Neurochir Suppl 102:53-55, 2008

38. Zabramski JM, Whiting D, Darouiche RO, Horner TG, Olson J, Robertson C, et al: Efficacy of antimicrobial-impregnated external ventricular drain catheters: a prospective, randomized, controlled trial. J Neurosurg 98:725-730, 2003

\section{Disclosures}

The authors report no conflict of interest concerning the materials or methods used in this study or the findings specified in this paper.

\section{Author Contributions}

Conception and design: Mounier, Birnbaum, Cook, Martin, Dhonneur. Acquisition of data: Mounier, Jost, Martin, Aït-Mamar, Nebbad, Couffin, Tomberli, Djedid. Analysis and interpretation of data: Mounier, Cook, Nebbad, Couffin, Tomberli, Djedid. Drafting the article: Mounier, Cook, Dhonneur. Critically revising the article: Mounier, Birnbaum, Cook, Dhonneur, Lobo. Approved the final version of the manuscript on behalf of all authors: Mounier. Statistical analysis: Birnbaum, Lobo. Administrative/technical/ material support: Mounier, Cook, Jost, Aït-Mamar, Lobo. Study supervision: Mounier, Cook.

\section{Correspondence}

Roman Mounier: Henri Mondor University Hospital, Créteil, France.roman.mounier@laposte.net. 